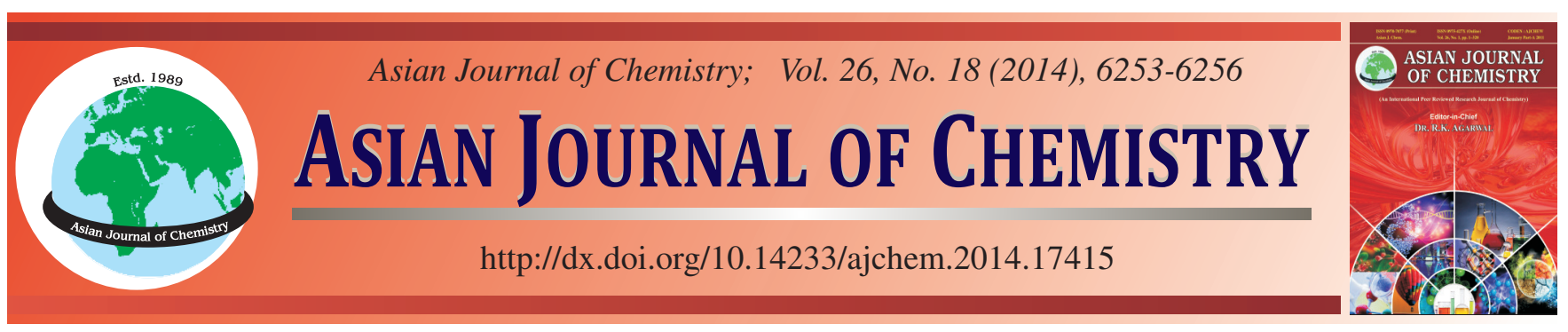

\title{
Synthesis, Antibacterial Activity and Antifouling Property of Novel Indole Compounds Containing Acylamino Groups
}

\author{
Xia Li, Chun-Hua Ni, Liang-Min Yu, Jing Zhao and Xue-Feng Yan*
}

Key Laboratory of Marine Chemistry Theory and Technology, Ministry of Education, Ocean University of China, Qingdao, P.R. China

*Corresponding author: Tel: +86 532 66782407; E-mail: yanxuefeng@ouc.edu.cn; xiali@ ouc.edu.cn

\begin{abstract}
Due to the environmentally harmful impact of tributyltin self-polishing paints and other "tributyltin-free" chemically active paints, there is a critical need of more ecological alternatives. In this work, 9 novel $\mathrm{N}$-substituted indoles each bearing a acylamino group were synthesized and the structures of $\mathbf{2 a - 2 i}$ were established by ${ }^{1} \mathrm{H}$ NMR, IR spectra and elemental analysis. The antibacterial activity of the synthesized compounds against Escherichia coli and Staphylococcus aureus as well as antifouling activity were studied. The results showed that these compounds possessed a broad spectrum of activity having MIC values of $0.01-0.5 \mathrm{mg} / \mathrm{mL}$ against the tested bacteria and antifouling property was superior to cuprous oxide which is widely used as antifoulants. This provided theoretical and technical support for the preparation of environmental friendly antifouling coatings.
\end{abstract}

Keywords: Indole, Acylamino groups, Synthesis, Antibacterial activity, Antifouling property.

\section{INTRODUCTION}

Since the ban on using tributyltin (TBT) ${ }^{1}$ as an antifouling agent on ship hulls and other marine facilities, the antifouling industry tried to utilize alternative "tributyltin-free" chemically active paint systems such as $\mathrm{Cu}_{2} \mathrm{O}$, Irgarol 1051 and chlorothalonil etc. ${ }^{2-4}$. However, these alternatives to tributyltin were also found to be toxic to other non-target organisms and thus the search for new antifouling agents which are non-toxic and biodegradable is necessary. The idea that searching for antifouling substances from secondary metabolites in the marine environment was first introduced in 1982 by Targett et $a .^{5}$ and the isolation and identification of molecules from marine organisms with antifouling activity ${ }^{6-8}$ had been reported by several studies. Among those bioactive metabolites, indole derivatives 2,5,6-tribromo-1-methylgramine (TBG) isolated from the bryozoan Zoobotryon pellucidum and 6-bromoindole3-carbaldehyde isolated from the ascidian Stomozoa murrayi had shown potent antifouling activity against barnacle larvae $\mathrm{e}^{9-10}$. Many researches focused on the synthesis of the analogues of 2,5,6-tribromo-1-methylgramine and 6-bromoindole-3carbaldehyde together with their biological activity by reason of their simple structure and high antifouling capability ${ }^{10-12}$.

In previous work, we reported the synthesis of $N$-substituted indoles each bearing a ester group and found these compounds especially which had a long chain ester group displayed high anti-algae activities against Nitzschia closterium $^{13}$. For the present study, dithiodipropionamides moiety was integrated into the scaffold of indole which has free $\mathrm{NH}$ group substituted by acylamino to generate series of new compounds (SchemeI). In addation, their antibacterial activity against Gram-positive and Gram-negative bacteria was assessed together with their antifouling property.

\section{EXPERIMENTAL}

Two bacteria of Staphyloccocus aureus (Gram-positive bacteria, ATCC 13565) and Escherichia coli (Gram-negative bacteria, ATCC 12435) were provided by the Lab of microorganism in the Ocean University of China. The bacteria were cultivated in biochemical incubator and prepared for the experiments. All solvents were dried in a routine way and redistilled. The chemical reagents were commercially available and treated with standard methods before using. Five dithiodipropionamides were prepared according to the previously reported method ${ }^{14}$. IR spectra were recorded for $\mathrm{KBr}$ disc on an AVATAR-360 FTIR spectrophotometer. ${ }^{1} \mathrm{H}$ NMR spectra were recorded with JUM-ECP $600(600 \mathrm{MHz})$ instrument in $\mathrm{CDCl}_{3}$ as solvent, using TMS as an internal standard and chemical shifts were expressed in parts per million $(\delta)$. Elemental analyses were performed by FLASH EA112 Analyzer and results for $\mathrm{C}, \mathrm{H}, \mathrm{N}$ were within $\pm 0.4 \%$ of calculated values.

General procedure: All the compounds were prepared as follows: Sodium hydride $(60 \%, 0.9 \mathrm{~g}, 18 \mathrm{mmol})$ was dispersed in anhydrous tetrahydrofuran $(15 \mathrm{~mL})$ at $0-5^{\circ} \mathrm{C}$ and 


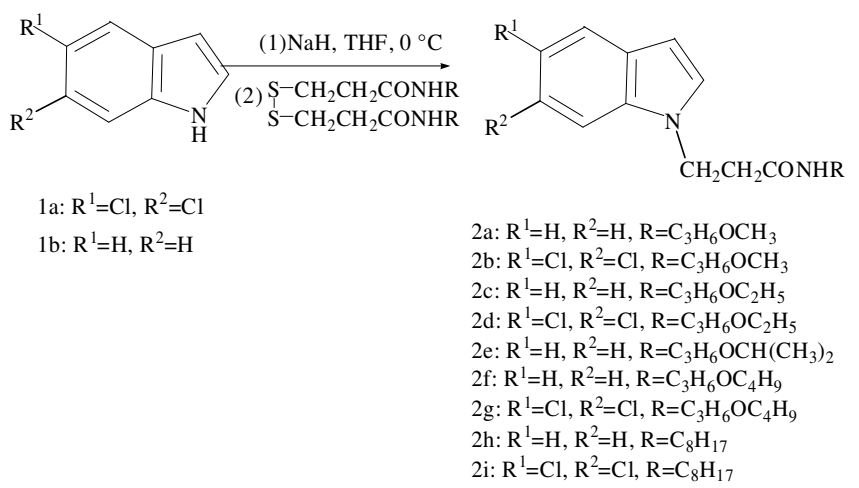

Scheme-I: Synthetic route of compounds $\mathbf{2 a - 2 i}$

compound 1a or $\mathbf{1 b}(10 \mathrm{mmol})$ was added successively. After stirring thoroughly for $0.5 \mathrm{~h}$, a solution of dithiodipro-pionamide $(10.5 \mathrm{mmol})$ in anhydrous tetrahydrofuran $(10 \mathrm{~mL})$ was added dropwise. The reaction mixture was stirred at room temperature for about $3.5 \mathrm{~h}$ and the reaction was quenched with saturated ammonium chloride solution. After extraction with ethyl acetate, the combined organic layers were washed with brine and dried over anhydrous $\mathrm{CaCl}_{2}$. After removal of the solvent in vacuo, the residue was purified by column chromatography with acetone as an eluent to afford $\mathbf{2 a - 2 i}$ with $32-53 \%$ yields.

3-(1H-indol-1-yl)-N-(3-methoxypropyl)propanamide (2a): Yellow oil; yield $53 \%$. ${ }^{1} \mathrm{H} \mathrm{NMR}\left(\mathrm{CDCl}_{3}, 600 \mathrm{MHz}\right)$ $\delta$ : $1.58\left(\mathrm{~m}, 2 \mathrm{H}, \mathrm{CH}_{2}\right), 2.57\left(\mathrm{t}, 2 \mathrm{H}, J=6.3 \mathrm{~Hz}, \mathrm{CH}_{2} \mathrm{CO}\right), 3.17$ (s, $\left.3 \mathrm{H}, \mathrm{CH}_{3}\right), 3.21\left(\mathrm{~m}, 2 \mathrm{H}, \mathrm{NHCH}_{2}\right), 3.25(\mathrm{t}, 2 \mathrm{H}, J=6.3 \mathrm{~Hz}$, $\left.\mathrm{CH}_{2} \mathrm{O}\right), 4.46\left(\mathrm{t}, 2 \mathrm{H}, J=6.4 \mathrm{~Hz}, \mathrm{NCH}_{2}\right), 5.83(\mathrm{~s}, 1 \mathrm{H}, \mathrm{NH})$, 6.45-7.60 (m, 6H, ArH). IR (KBr, $\left.v_{\max }, \mathrm{cm}^{-1}\right)$ : 742.3, 1210.3, 1223.4, 1646.7, 2876.8, 2933.8, 3292.3. For $\mathrm{C}_{15} \mathrm{H}_{20} \mathrm{~N}_{2} \mathrm{O}_{2}$ $\left(\mathrm{M}_{\mathrm{r}}=260.33\right) \mathrm{w}_{\mathrm{i}}$ (calc. $) / \%: \mathrm{C} 69.20, \mathrm{H} 7.74, \mathrm{~N} 10.76 ; \mathrm{w}_{\mathrm{i}}$ (found)/\%: C 69.27, H 7.70, N 10.72.

3-(5,6-dichloro-1H-indol-1-yl)-N-(3-methoxypropyl)propanamide (2b): Brown oil; yield $32 \%$. ${ }^{1} \mathrm{H} \mathrm{NMR}\left(\mathrm{CDCl}_{3}\right.$, $600 \mathrm{MHz}) \delta: 1.61\left(\mathrm{~m}, 2 \mathrm{H}, \mathrm{CH}_{2}\right), 2.59(\mathrm{t}, 2 \mathrm{H}, J=6.5 \mathrm{~Hz}$, $\mathrm{CH}_{2} \mathrm{CO}$ ), 3.24 (s, $\left.3 \mathrm{H}, \mathrm{CH}_{3}\right), 3.30\left(\mathrm{~m}, 2 \mathrm{H}, \mathrm{NHCH}_{2}\right), 3.34$ (t, $\left.2 \mathrm{H}, J=6.3 \mathrm{~Hz}, \mathrm{CH}_{2} \mathrm{O}\right), 4.42\left(\mathrm{t}, 2 \mathrm{H}, J=6.5 \mathrm{~Hz}, \mathrm{NCH}_{2}\right), 6.37$ (s, 1H, NH), 7.13-7.65 (m, 4H, ArH). IR (KBr, $\left.v_{\max }, \mathrm{cm}^{-1}\right)$ : 717.5, 1191.6, 1250.6, 1645.0, 2871.1, 2927.9, 3291.3. For $\mathrm{C}_{15} \mathrm{H}_{18} \mathrm{~N}_{2} \mathrm{O}_{2} \mathrm{Cl}_{2}\left(\mathrm{M}_{\mathrm{r}}=329.22\right) \mathrm{w}_{\mathrm{i}}($ calc. $) / \%$ : C 54.72, H 5.51, N 8.51; $\mathrm{w}_{\mathrm{i}}$ (found)/\%: C 54.66, H 5.53, N 8.54.

3-(1H-indol-1-yl)-N-(3-ethoxypropyl)propanamide (2c): Orange oil; yield $48 \% .{ }^{1} \mathrm{H} \mathrm{NMR}\left(\mathrm{CDCl}_{3}, 600 \mathrm{MHz}\right) \delta$ : 1.07 (t, 3H, $\left.J=7.2 \mathrm{~Hz}, \mathrm{CH}_{3}\right), 1.60\left(\mathrm{~m}, 2 \mathrm{H}, \mathrm{CH}_{2}\right), 2.58(\mathrm{t}, 2 \mathrm{H}$, $\left.J=6.6 \mathrm{~Hz}, \mathrm{CH}_{2} \mathrm{CO}\right), 3.24\left(\mathrm{~m}, 2 \mathrm{H}, \mathrm{NHCH}_{2}\right), 3.29(\mathrm{~m}, 2 \mathrm{H}$, $\left.\mathrm{OCH}_{2} \mathrm{CH}_{3}\right), 3.32\left(\mathrm{t}, 2 \mathrm{H}, J=6.5 \mathrm{~Hz}, \mathrm{CH}_{2} \mathrm{O}\right), 4.49(\mathrm{t}, 2 \mathrm{H}, J=$ $6.4 \mathrm{~Hz}, \mathrm{NCH}_{2}$ ), 5.93 (s, 1H, NH), 6.46-7.61(m, 6H, ArH). IR $\left(\mathrm{KBr}, v_{\max }, \mathrm{cm}^{-1}\right)$ : 741.7, 1219.0, 1646.6, 2866.8, 2973.8, 3292.3. For $\mathrm{C}_{16} \mathrm{H}_{22} \mathrm{~N}_{2} \mathrm{O}_{2}\left(\mathrm{M}_{\mathrm{r}}=274.36\right) \mathrm{w}_{\mathrm{i}}$ (calc.)/\%: C 70.04, $\mathrm{H} 8.08, \mathrm{~N} 10.21$; $\mathrm{w}_{\mathrm{i}}$ (found)/\%: C 70.09, H 8.03, N 10.23.

3-(5,6-dichloro-1H-indol-1-yl)-N-(3-ethoxypropyl)propanamide (2d): Brown oil; yield $38 \%$. ${ }^{1} \mathrm{H} \mathrm{NMR}\left(\mathrm{CDCl}_{3}\right.$, $600 \mathrm{MHz}) \delta: 1.07$ (t, 3H, J=3.5 Hz, $\left.\mathrm{CH}_{3}\right), 1.59\left(\mathrm{~m}, 2 \mathrm{H}, \mathrm{CH}_{2}\right)$, $2.54\left(\mathrm{t}, 2 \mathrm{H}, J=6.5 \mathrm{~Hz}, \mathrm{CH}_{2} \mathrm{CO}\right), 3.22\left(\mathrm{~m}, 2 \mathrm{H}, \mathrm{NHCH}_{2}\right), 3.29$ $\left(\mathrm{m}, 2 \mathrm{H}, \mathrm{OCH}_{2} \mathrm{CH}_{3}\right), 3.33\left(\mathrm{t}, 2 \mathrm{H}, J=3.7, \mathrm{CH}_{2} \mathrm{O}\right), 4.37$ (t, $2 \mathrm{H}, J$ $\left.=3.5 \mathrm{~Hz}, \mathrm{NCH}_{2}\right), 6.36(\mathrm{~s}, 1 \mathrm{H}, \mathrm{NH}), 6.46-7.64(\mathrm{~m}, 4 \mathrm{H}, \mathrm{ArH})$.
IR $\left(\mathrm{KBr}, v_{\max }, \mathrm{cm}^{-1}\right):$ 716.0, 1217.0, 1217.8, 1644.6, 2865.7, 2929.2, 3297.8. For $\mathrm{C}_{16} \mathrm{H}_{20} \mathrm{~N}_{2} \mathrm{O}_{2} \mathrm{Cl}_{2}\left(\mathrm{M}_{\mathrm{r}}=343.25\right) \mathrm{w}_{\mathrm{i}}$ (calc.)/ $\%$ : C 55.99, H 5.87, N 8.16; $\mathrm{w}_{\mathrm{i}}$ (found)/\%: C 55.94, H 5.84, N 8.20 .

3-(1H-indol-1-yl)-N-(3-isopropoxypropyl)-propanamide (2e): Orange oil; yield $39 \%$. ${ }^{1} \mathrm{H} \mathrm{NMR}\left(\mathrm{CDCl}_{3}, 600 \mathrm{MHz}\right)$ $\delta: 1.03\left(\mathrm{~d}, 6 \mathrm{H}, J=6.4 \mathrm{~Hz}, \mathrm{CH}\left(\mathrm{CH}_{3}\right)_{2}\right), 1.58\left(\mathrm{~m}, 2 \mathrm{H}, \mathrm{CH}_{2}\right)$, 2.57 (t, 2H, $\left.J=6.6 \mathrm{~Hz}, \mathrm{CH}_{2} \mathrm{CO}\right), 3.24\left(\mathrm{~m}, 2 \mathrm{H}, \mathrm{NHCH}_{2}\right), 3.33$ $\left(\mathrm{t}, 2 \mathrm{H}, J=5.4 \mathrm{~Hz}, \mathrm{CH}_{2} \mathrm{O}\right), 3.41(\mathrm{~m}, 1 \mathrm{H}, \mathrm{CH}), 4.47(\mathrm{t}, 2 \mathrm{H}, J=$ $\left.6.4 \mathrm{~Hz}, \mathrm{NCH}_{2}\right), 6.11$ (s, 1H, NH), 6.45-7.60 (m, 6H, ArH). IR $\left(\mathrm{KBr}, v_{\max }, \mathrm{cm}^{-1}\right): 741.3,1233.41,1648.0,2872.2,2970.7$, 3302.4. For $\mathrm{C}_{17} \mathrm{H}_{24} \mathrm{~N}_{2} \mathrm{O}_{2}\left(\mathrm{M}_{\mathrm{r}}=288.38\right) \mathrm{w}_{\mathrm{i}}$ (calc.)/\%: C 70.80, H 8.39, N 9.71; $\mathrm{w}_{\mathrm{i}}$ (found)/\%: C 70.69, H 8.47, N 9.73.

3-(1H-indol-1-yl)-N-(3-butoxypropyl) propanamide (2f): Orange oil; yield $42 \% .{ }^{1} \mathrm{H} \mathrm{NMR}\left(\mathrm{CDCl}_{3}, 600 \mathrm{MHz}\right) \delta$ : 0.88 (t, $\left.3 \mathrm{H}, J=7.2 \mathrm{~Hz}, \mathrm{CH}_{3}\right), 1.28\left(\mathrm{~m}, 2 \mathrm{H}, \mathrm{C}_{4} \mathrm{H}_{9}, 2-\mathrm{H}\right), 1.43$ $\left(\mathrm{m}, 2 \mathrm{H}, \mathrm{C}_{4} \mathrm{H}_{9}, 3-\mathrm{H}\right), 1.60\left(\mathrm{~m}, 2 \mathrm{H}, \mathrm{CH}_{2}\right), 2.58(\mathrm{t}, 2 \mathrm{H}, J=6.6$ $\left.\mathrm{Hz}, \mathrm{CH}_{2} \mathrm{CO}\right), 3.26\left(\mathrm{~m}, 2 \mathrm{H}, \mathrm{NHCH}_{2}\right), 3.27$ (t, 2H, $J=6.6 \mathrm{~Hz}$, $\left.\mathrm{OCH}_{2}\right), 3.33\left(\mathrm{t}, 2 \mathrm{H}, J=6.4 \mathrm{~Hz}, \mathrm{CH}_{2} \mathrm{O}\right), 4.49(\mathrm{t}, 2 \mathrm{H}, J=6.6 \mathrm{~Hz}$, $\left.\mathrm{NCH}_{2}\right), 5.94(\mathrm{~s}, 1 \mathrm{H}, \mathrm{NH}), 6.45-7.61(\mathrm{~m}, 6 \mathrm{H}, \mathrm{ArH})$. IR (KBr, $\left.V_{\max }, \mathrm{cm}^{-1}\right)$ : 741.3, 1080.9, 1664.6, 2871.8, 2957.8, 3308.5. For $\mathrm{C}_{18} \mathrm{H}_{26} \mathrm{~N}_{2} \mathrm{O}_{2}\left(\mathrm{M}_{\mathrm{r}}=302.41\right) \mathrm{w}_{\mathrm{i}}$ (calc.)/\%: C 71.49, H 8.67, $\mathrm{N} 9.26 ; \mathrm{w}_{\mathrm{i}}$ (found)/\%: C 71.45, H 8.69, N 9.26.

3-(5,6-dichloro-1H-indol-1-yl)-N-(3-butoxypropyl)propanamide (2g): Brown oil; yield $36 \%$. ${ }^{1} \mathrm{H} \mathrm{NMR}\left(\mathrm{CDCl}_{3}\right.$, $600 \mathrm{MHz}) \delta: 0.88\left(\mathrm{t}, 3 \mathrm{H}, J=7.2 \mathrm{~Hz}, \mathrm{CH}_{3}\right), 1.30\left(\mathrm{~m}, 2 \mathrm{H}, \mathrm{C}_{4} \mathrm{H}_{9}\right.$, 2-H), 1.45 (m, 2H, $\left.\mathrm{C}_{4} \mathrm{H}_{9}, 3-\mathrm{H}\right), 1.58\left(\mathrm{~m}, 2 \mathrm{H}, \mathrm{CH}_{2}\right), 2.59$ (t, 2H, $\left.J=6.6 \mathrm{~Hz}, \mathrm{CH}_{2} \mathrm{CO}\right), 3.26\left(\mathrm{~m}, 2 \mathrm{H}, \mathrm{NHCH}_{2}\right), 3.27(\mathrm{t}, 2 \mathrm{H}, J=$ $\left.6.6 \mathrm{~Hz}, \mathrm{OCH}_{2}\right), 3.33$ (t, $\left.2 \mathrm{H}, J=6.4 \mathrm{~Hz}, \mathrm{CH}_{2} \mathrm{O}\right), 4.49$ (t, $2 \mathrm{H}$, $\left.J=6.6 \mathrm{~Hz}, \mathrm{NCH}_{2}\right), 6.03(\mathrm{~s}, 1 \mathrm{H}, \mathrm{NH}), 6.45-7.59$ (m, 4H, ArH). IR $\left(\mathrm{KBr}, \mathrm{v}_{\max }, \mathrm{cm}^{-1}\right): 759.9,1249.9,1653.5,2866.9,2956.7$, 3282.8. For $\mathrm{C}_{18} \mathrm{H}_{24} \mathrm{~N}_{2} \mathrm{O}_{2} \mathrm{Cl}_{2}\left(\mathrm{M}_{\mathrm{r}}=371.30\right) \mathrm{W}_{\mathrm{i}}$ (calc.)/\%: C 58.23, H 6.52, N 7.54; $\mathrm{w}_{\mathrm{i}}$ (found)/\%: C 58.20, H 6.51, N 7.55.

3-(1H-indol-1-yl)-N-octylpropanamide (2h): Brown oil; yield $39 \% .{ }^{1} \mathrm{H} \mathrm{NMR}\left(\mathrm{CDCl}_{3}, 600 \mathrm{MHz}\right) \delta: 0.87(\mathrm{t}, 3 \mathrm{H}, J=7.2$ $\left.\mathrm{Hz}, \mathrm{CH}_{3}\right), 1.13\left(\mathrm{~m}, 2 \mathrm{H}, \mathrm{C}_{8} \mathrm{H}_{17}, 2-\mathrm{H}\right), 1.20\left(\mathrm{~m}, 2 \mathrm{H}, \mathrm{C}_{8} \mathrm{H}_{17}, 3-\mathrm{H}\right)$, $1.20\left(\mathrm{~m}, 2 \mathrm{H}, \mathrm{C}_{8} \mathrm{H}_{17}, 4-\mathrm{H}\right), 1.20\left(\mathrm{~m}, 2 \mathrm{H}, \mathrm{C}_{8} \mathrm{H}_{17}, 5-\mathrm{H}\right), 1.24(\mathrm{~m}$, $\left.2 \mathrm{H}, \mathrm{C}_{8} \mathrm{H}_{17}, 6-\mathrm{H}\right), 1.24\left(\mathrm{~m}, 2 \mathrm{H}, \mathrm{C}_{8} \mathrm{H}_{17}, 7-\mathrm{H}\right), 2.57$ (t, $2 \mathrm{H}, J=6.6$ $\mathrm{Hz}, \mathrm{CH}_{2} \mathrm{CO}$ ), 3.08 (t, 2H, J = 9.6 Hz, $\mathrm{NHCH}_{2}$ ), 4.47 (t, $2 \mathrm{H}$, $\left.J=6.3 \mathrm{~Hz}, \mathrm{NCH}_{2}\right), 5.20$ (s, 1H, NH), 6.43-7.59 (m, 6H, ArH). IR $\left(\mathrm{KBr}, v_{\max }, \mathrm{cm}^{-1}\right): 735.5,1238.9,1456.8,1556.8,1659.8$, 2922.6, 3287.6. For $\mathrm{C}_{19} \mathrm{H}_{28} \mathrm{~N}_{2} \mathrm{O}\left(\mathrm{M}_{\mathrm{r}}=300.44\right) \mathrm{w}_{\mathrm{i}}$ (calc.)/\%: C 75.96, H 9.39, N 9.32; $\mathrm{w}_{\mathrm{i}}$ (found)/\%: C 75.99, H 9.38, N 9.30.

3-(5,6-dichloro-1H-indol-1-yl)-N-octylpropanamide (2i): Red oil; yield $36 \% .{ }^{1} \mathrm{H} \mathrm{NMR}\left(\mathrm{CDCl}_{3}, 600 \mathrm{MHz}\right) \delta: 0.89$ (t, 3H, J = 7.2 Hz, $\mathrm{CH}_{3}$ ), $1.10\left(\mathrm{~m}, 2 \mathrm{H}, \mathrm{C}_{8} \mathrm{H}_{17}, 2-\mathrm{H}\right), 1.20$ (m, $\left.2 \mathrm{H}, \mathrm{C}_{8} \mathrm{H}_{17}, 3-\mathrm{H}\right), 1.20$ (m, 2H, $\left.\mathrm{C}_{8} \mathrm{H}_{17}, 4-\mathrm{H}\right), 1.20$ (m, 2H, $\mathrm{C}_{8} \mathrm{H}_{17}$, 5-H), 1.30 (m, 2H, $\left.\mathrm{C}_{8} \mathrm{H}_{17}, 6-\mathrm{H}\right), 1.30$ (m, 2H, $\left.\mathrm{C}_{8} \mathrm{H}_{17}, 7-\mathrm{H}\right)$, $2.56\left(\mathrm{t}, 2 \mathrm{H}, J=6.3 \mathrm{~Hz}, \mathrm{CH}_{2} \mathrm{CO}\right), 3.10(\mathrm{t}, 2 \mathrm{H}, J=9.6 \mathrm{~Hz}$, $\left.\mathrm{NHCH}_{2}\right), 4.40$ (t, $\left.2 \mathrm{H}, J=6.3 \mathrm{~Hz}, \mathrm{NCH}_{2}\right), 5.45$ (s, 1H, NH), 6.35-7.65 (m, 4H, ArH). IR (KBr, $\left.v_{\max }, \mathrm{cm}^{-1}\right)$ : 717.9, 1115.3, 1465.6, 1556.8, 1639.2, 2919.71 3287.6. For $\mathrm{C}_{19} \mathrm{H}_{26} \mathrm{~N}_{2} \mathrm{OCl}_{2}$ $\left(\mathrm{M}_{\mathrm{r}}=369.33\right) \mathrm{w}_{\mathrm{i}}($ calc. $) / \%$ : C 61.79, H 7.10, N 7.58; $\mathrm{w}_{\mathrm{i}}$ (found)/ $\%$ : C 61.75, H 7.12, N 7.55.

\section{Antibacterial activity test}

Cultivation of bacteria: Staphyloccocus aureus and Escherichia coli were transferred to the basal media (peptone: $10 \mathrm{~g}$, beef extract: $3 \mathrm{~g}, \mathrm{NaCl}: 50 \mathrm{~g}, \mathrm{Na}_{2} \mathrm{HPO}_{4} \cdot 12 \mathrm{H}_{2} \mathrm{O}: 9 \mathrm{~g}$, 
$\mathrm{KH}_{2} \mathrm{PO}_{4}: 1.5 \mathrm{~g}$, distilled water: $1000 \mathrm{~mL}$, $\mathrm{pH} 7.2$, sterilization for ten minutes at $121^{\circ} \mathrm{C}$ ) ${ }^{15}$ from the fridge, cultivated for $24 \mathrm{~h}$ at $37 \pm 1{ }^{\circ} \mathrm{C}$ and then single bacterial colony was obtained. Bacteria were scraped from the culture plate and resuspended in $5 \mathrm{~mL}$ liquid nutrient medium and the normal saline was added to the liquid nutrient medium at the desired final concentration for the two-fold serial dilution.

Determination of antibacterial activity: The assays were performed as two-fold serial dilution tests for the determination of the minimum inhibition concentration (MIC) ${ }^{16}$. Every test tube had $2 \mathrm{~mL}$ nutrient fluid medium and the initial concentration of tested compound was $1 \mathrm{mg} / \mathrm{mL}$ acetone, double dilution in turns followed by vortex mixing. One test tube contained bacteria without sample (growth control) and another one only contained liquid nutrient medium (sterility control). Then definite concentration (104-105 colony forming units per $\mathrm{mL}$ ) of the bacterium solution were put into test tubes. Finally, exponentially growing cultures were incubated with increasing doses of nine tested compounds in the range of 0 to $1 \mathrm{mg} / \mathrm{mL}$ at $37{ }^{\circ} \mathrm{C}$ for $24 \mathrm{~h}$ in suspension in liquid nutrient medium. All tests were conducted in triplicate.

in situ Evaluation: Six new compounds were selected as antifoulants in the preparation of antifouling paints. Paints were formulated by dispersing all the ingredients (Table-1) with mechanical agitator under vigorous agitation $(2000 \mathrm{rpm})$ for $1 \mathrm{~h}$. Then the paints were filtered through a sifter $(100 \mu \mathrm{m})$.

Antifouling property of paints was investigated according to GB 5370-85 $5^{17}$. Paints were applied onto the low carbon steel plates $(250 \mathrm{~mm} \times 150 \mathrm{~mm} \times 3 \mathrm{~mm})$ after being polished and descaled with sandpaper. A commercial paint with cuprous oxide as antifoulant was used as a performance reference. Nonbiocidal paint was used as negative control. Panels were immersed $1 \mathrm{~m}$ deep in the Yellow Sea (Qingdao Harbour, China, $36^{\circ}$ $\left.04^{\prime} \mathrm{N}, 120^{\circ} 19^{\prime} 05^{\prime \prime} \mathrm{E}\right)$. And the marine coating systems were exposed in vertical position to sea water for 4 months (from June to September) when marine alga and barnacle represented vigorous growth in this period.

\section{RESULTS AND DISCUSSION}

Synthesis of compounds: The general synthetic route of

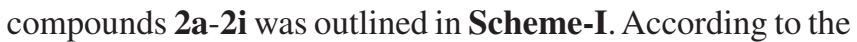
literature ${ }^{18}$, compounds $1 \mathrm{a}$ and $1 \mathrm{~b}$ were reacted with $\mathrm{NaH}$ in dry THF or N,N-dimethyl formamide (DMF) under lower temperature to afford heterocycle anion, then followed by Michael addition with dithiodipropionamide, which generated amide and served in situ as a source of propionamide moiety to give the target compounds $\mathbf{2 a - 2 i}$ in yields of $32-53 \%$. The experimental results indicated that the solvent determined the yields of title compounds. For $\mathbf{2 a - 2 g}$, regardless selecting DMF or THF as the solvent, the generation rates of compounds were similar, but the yields was lower when selecting DMF as the solvent because of its good solubility in water. For $\mathbf{2 h}-\mathbf{2} \mathbf{i}$, which were obtained by using DMF as the solvent with good yields, but no product was observed when using THF as the solvent under the same condition. Furthermore, the reaction time was found to vary from 3 to $5 \mathrm{~h}$ depending upon the substitutions on the acylamino group.

The appearance of $\mathrm{C}=\mathrm{O}$ band at $1664.6-1639.2 \mathrm{~cm}^{-1}$ region and $\mathrm{N}-\mathrm{H}$ band at $3308.5-3282.8 \mathrm{~cm}^{-1}$ region in the IR spectra of isolated products and the lack of signals due to $N-1$ proton of indole ring in their ${ }^{1} \mathrm{H}$ NMR spectra provided a firm support for novel compounds $\mathbf{2 a - 2 i}$.

MIC of compounds: The MIC values of nine compounds against Staphyloccocus aureus and Escherichia coli were shown in Table-2.

\begin{tabular}{ccc}
\multicolumn{3}{c}{ TABLE-2 } \\
MIC OF COMPOUNDS AGAINST SELECTED BACTERIA \\
\hline \multirow{2}{*}{ Compound } & \multicolumn{2}{c}{ MIC $\left(\mathrm{mg} \cdot \mathrm{mL}^{-1}\right)$} \\
\cline { 2 - 3 } & Escherichia coli & Staphyloccocus aureus \\
\hline $\mathbf{2 a}$ & 0.50 & 0.12 \\
$\mathbf{2 b}$ & 0.50 & 0.06 \\
$\mathbf{2} \mathbf{c}$ & 0.50 & 0.06 \\
$\mathbf{2 d}$ & 0.25 & 0.03 \\
$\mathbf{2 e}$ & 0.25 & 0.03 \\
$\mathbf{2 f}$ & 0.25 & 0.06 \\
$\mathbf{2 g}$ & 0.12 & 0.01 \\
$\mathbf{2 h}$ & 0.06 & 0.06 \\
$\mathbf{2} \mathbf{i}$ & 0.03 & 0.06 \\
Tributyltin oxide & 1.00 & 0.50 \\
\hline
\end{tabular}

The listed MIC values in Table-2 showed that all the synthesized compounds had profound antibacterial potency as compared to the reference antifoulant tributyltin oxide within a MIC range of $0.01-0.50 \mathrm{mg} / \mathrm{mL}$. And compounds showed higher antibacterial activities against Staphyloccocus aureus than against Escherichia coli except for $\mathbf{2 i}$ which showed moderate activity $(0.06 \mathrm{mg} / \mathrm{mL})$ against Staphyloccocus aureus but significant activity against Escherichia coli $(0.03 \mathrm{mg} / \mathrm{mL})$. In comparison, the antibacterial results of the compounds followed the order of: $\mathbf{2 h}>\mathbf{2 f}>\mathbf{2 e}>\mathbf{2 c}>\mathbf{2 a}$ and $\mathbf{2 i}>\mathbf{2 g}>\mathbf{2 d}$ $>\mathbf{2 b}$, which suggested that long alkyl chain attached to the moiety enhanced the lipophilic activity and increased the biological activity of these compounds. In addition, it was observed that halogen as electron-withdrawing groups in indole ring tended to enhance the antibacterial activity, for example,

\begin{tabular}{|c|c|c|c|c|c|c|}
\hline \multicolumn{7}{|c|}{$\begin{array}{c}\text { TABLE-1 } \\
\text { COMPOSITION OF ANTIFOULING PAINTS }^{a}\end{array}$} \\
\hline No. & Acrylic resin & Cuprous oxide & Pigment & Assistant antifoulant & Assistant & Xylene \\
\hline 1 & 55 & 25 & 17 & $10(2 c)$ & 2 & 11 \\
\hline 2 & 55 & 25 & 17 & $10(2 d)$ & 2 & 11 \\
\hline 3 & 55 & 25 & 17 & $10(2 \mathrm{f})$ & 2 & 12 \\
\hline 4 & 55 & 25 & 17 & $10(2 \mathrm{~g})$ & 2 & 11 \\
\hline 5 & 55 & 25 & 17 & $10(2 \mathrm{~h})$ & 2 & 12 \\
\hline 6 & 55 & 25 & 17 & $10(2 \mathrm{i})$ & 2 & 11 \\
\hline 7 & 55 & 25 & 17 & 10 (cuprous oxide) & 2 & 11 \\
\hline
\end{tabular}


the anti-Staphyloccocus aureus activity of compound $\mathbf{2 g}(0.01$ $\mathrm{mg} \times \mathrm{mL}^{-1}$ ) was obviously higher than that of compound $\mathbf{2 f}$ $\left(0.06 \mathrm{mg} \times \mathrm{mL}^{-1}\right)$.

Antifouling properties: Antifouling paints containing active substances were immersed in natural seawater. The biofouling were inspected and photographed at intervals and the results were shown in Table-3.

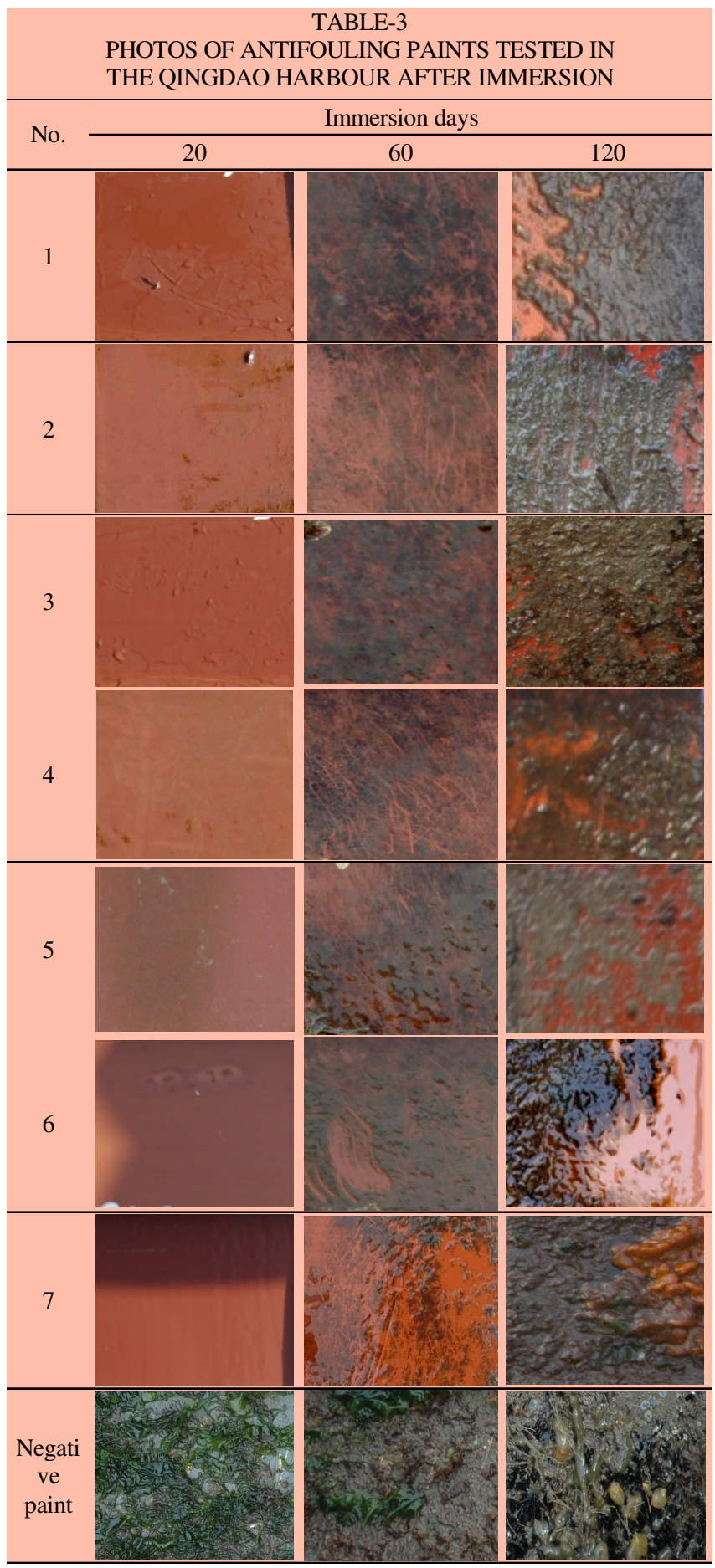

After 20 days of immersion, a well established biofilm was observed on unloaded varnish and large numbers of green algae were also present. There is no observation on any marine organisms on the other paints. After 60 days, all paints were covered with biofilm together with some biological sludge except for the negative paint, on which many biological sludge and a few marine animals were found. Paints $\mathbf{2}$ and 4 had less biofilm on the surface than paints $\mathbf{1}$ and $\mathbf{3}$ respectively which displayed that indole derivatives synthesized in this article containing chlorine showed superior antifouling activity and it was also displayed synthesized compounds had no advantage than cuprous oxide in inhibiting adhesion of marine organisms by comparing paints 1-6 to paint $\mathbf{7}$ in these days. Over a stretch of 120 days, there were more macroorganisms on the negative paint such as mussels and calcarina and big animal were also found the paint 7 which were already covered by biofilm and brown algae fully. Fortunately, we didn't see any macroorganisms but only biofilm and microorganisms on the paints from $\mathbf{1}$ to $\mathbf{6}$, which means the antifouling property of new compounds is better than cuprous oxide from a long-term perspective and have good application prospect in antifouling paints.

\section{Conclusion}

The synthesis of nine novel indole derivatives that each contained a acylamino group had been achieved and their antibacterial activity against Escherichia coli and Staphylococcus aureus as well as antifouling property in the antifouling paints were studied. All synthesized compounds especially which had a long alkyl chain exhibited extremely high antifouling activities.

\section{ACKNOWLEDGEMENTS}

This work was financially supported by the NSFC (No. 51003099), "863" Program (Nos. 2010AA09Z203, 2010AA065104) and Doctoral Fund of Ministry of Education of China (No. 20130132130001).

\section{REFERENCES}

1. M.A. Champ, Mar. Pollut. Bull., 46, 935 (2003).

2. D.E. Moreland, Annu. Rev. Plant Physiol., 31, 597 (1980).

3. A. Koutsaftis and I. Aoyama, Sci. Total Environ., 387, 166 (2007).

4. F. Cima, M. Bragadin and L. Ballarin, Aquat. Toxicol., 86, 299 (2008).

5. N.M. Targett, S.S. Bishop, O.J. McConnell and J.A. Yoder, J. Chem. Ecol., 9, 817 (1983).

6. M. Sjögren, U. Göransson, A.L. Johnson, M. Dahlström, R. Andersson, J. Bergman, P.R. Jonsson and L. Bohlin, J. Nat. Prod., 67, 368 (2004).

7. N.A. Paul, R. de Nys and P.D. Steinberg, Mar. Ecol. Prog. Ser., 306, 87 (2006).

8. A. Bazes, A. Silkina, D. Defer, C. Bernède-Bauduin, E. Quéméner, J.P. Braud and N. Bourgougnon, Aquaculture, 258, 664 (2006).

9. H. Morii, Y. Izumi, K. Kasama and R. Ishimoto, Fish. Sci., 60, 773 (1994).

10. G. Olguin-uribe, E. Abou-mansour, A. Boulander, H. Débard, C. Francisco and G. Combaut, J. Chem. Ecol., 23, 2507 (1997).

11. N. Yamaguchi, K. Matsuzawa, M. Kono et al., JP Patent 2002053410 (2002).

12. Y. Shizuri, J. Tanaka, J. Fukunaga et al., JP Patent 2003055112 (2003).

13. X. Li, L.M. Yu, X.H. Jiang, S. Xia and H. Zhao, Chin. J. Oceanology Limnol., 27, 309 (2009).

14. W.W. Cong, L.M. Yu, X.H. Jiang, X. Li and H.Z. Zhao, Materials Rev., 25, 37 (2011)

15. F.S. Chen and Y.P. Xu, CN Patent 101045909 (2007).

16. W.W. Cong and L.M. Yu, Chem. Res. Chin. Univ., 27, 803 (2011).

17. Antifouling Panels in Shallow Submergence, Patent GB/T 5370-85.

18. P. Hamel and M. Girard, J. Org. Chem., 65, 3123 (2000). 